\title{
NON-COHEN-MACAULAY SYMBOLIC BLOW-UPS FOR SPACE MONOMIAL CURVES
}

\author{
MAYUMI MORIMOTO AND SHIRO GOTO
}

(Communicated by Louis J. Ratliff, Jr.)

\begin{abstract}
Let $\mathfrak{p}=\mathfrak{p}\left(n_{1}, n_{2}, n_{3}\right)$ denote the prime ideal in the formal power series ring $A=k[[X, Y, Z]]$ over a field $k$ defining the space monomial curve $X=T^{n_{1}}, Y=T^{n_{2}}$, and $Z=T^{n_{3}}$ with $\operatorname{GCD}\left(n_{1}, n_{2}, n_{3}\right)=1$. Then the symbolic Rees algebra $R_{s}(\mathfrak{p})=\bigoplus_{n \geq 0} \mathfrak{p}^{(n)}$ for $\mathfrak{p}=\mathfrak{p}\left(n^{2}+2 n+2, n^{2}+2 n+\right.$ $\left.1, n^{2}+n+1\right)$ is Noetherian but not Cohen-Macaulay if $\operatorname{ch} k=p>0$ and $n=p^{e}$ with $e \geq 1$. The same is true for $\mathfrak{p}=\mathfrak{p}\left(n^{2}, n^{2}+1, n^{2}+n+1\right)$ if $\operatorname{ch} k=p>0$ and $n=p^{e} \geq 3$.
\end{abstract}

\section{INTRODUCTION}

Let $A$ be a regular local ring of $\operatorname{dim} A=3$ and $\mathfrak{p}$ a prime ideal of $A$ with $\operatorname{dim} A / \mathfrak{p}=1$. Let $R_{S}(\mathfrak{p})=\sum_{n \geq 0} \mathfrak{p}^{(n)} t^{n}$ be the symbolic Rees algebra of $\mathfrak{p}$ (here $t$ denotes an indeterminate over $A$ ). In this situation Goto, Nishida, and Shimoda [2] investigated the conditions under which the symbolic Rees algebra $R_{s}(\mathfrak{p})$ is a Gorenstein ring. They gave a criterion for the case in terms of the elements $f$ and $g$ of $\mathfrak{p}$ in Huneke's condition [5] of $R_{s}(\mathfrak{p})$ being Noetherian and showed that $R_{S}(\mathfrak{p})$ are necessarily Gorenstein for a certain class of prime ideals $\mathfrak{p}$ (cf. $\S 2$ ).

The problem when $R_{s}(\mathfrak{p})$ is Noetherian was raised by Cowsik [1] and is more fundamental. However there is an example $\mathfrak{p}$ due to Roberts [7] (passing to Nagata's counterexamples [6] to the 14th problem of Hilbert) whose symbolic Rees algebra $R_{s}(\mathfrak{p})$ is not Noetherian, while Cowsik's problem is still open for the general prime ideals $\mathfrak{p}=\mathfrak{p}\left(n_{1}, n_{2}, n_{3}\right)$ in the formal power series ring $A=k[[X, Y, Z]]$ over a field $k$ defining space monomial curves $X=T^{n_{1}}$, $Y=T^{n_{2}}$, and $Z=T^{n_{3}}$ with $\operatorname{GCD}\left(n_{1}, n_{2}, n_{3}\right)=1$.

Throughout the research [2] of Goto, Nishida, and Shimoda they seemed to follow a natural conjecture that $R_{s}(\mathfrak{p})$ has to be a Gorenstein ring once it is Noetherian for the space monomial curves $\mathfrak{p}=\mathfrak{p}\left(n_{1}, n_{2}, n_{3}\right)$. We note here that $R_{s}(\mathfrak{p})$ is Gorenstein if it is Cohen-Macaulay (cf. [9, (3.4)]). Therefore the above conjecture is equivalent to saying that $R_{s}(\mathfrak{p})$ is always Cohen-Macaulay if it is Noetherian. Nevertheless this is not true in general, as we claim in the following

Received by the editors August 8, 1990 and, in revised form, March 13, 1991.

1991 Mathematics Subject Classification. Primary 13H10, 13H15; Secondary 13 E05.

The authors are partially supported by Grant-in-Aid for Co-operative Research. 
Theorem 1.1. Let $p$ be a prime number and $n=p^{e}$ with $e \geq 1$. Then $R_{s}(\mathfrak{p})$ is Noetherian but not Cohen-Macaulay for $\mathfrak{p}=\mathfrak{p}\left(n^{2}+2 n+2, n^{2}+2 n+1, n^{2}+n+1\right)$ if $\operatorname{ch} k=p$.

If we choose $n=p=2$ in Theorem 1.1 , then we have $\mathfrak{p}=\mathfrak{p}(10,9,7)$, which was already cited in $[2,(5.1)]$ too, while Vasconcelos $[10,4.1 .4]$ gave the defining equations of $R_{s}(\mathfrak{p})$ for $\mathfrak{p}=\mathfrak{p}(10,9,7)$ claiming its Gorensteinness. The disparity between the authors and Vasconcelos is caused by his implicit assumption that $\operatorname{ch} k=0$ and quite interesting: the Gorensteinness of symbolic Rees algebras depends on the characteristic of the ground field.

Similarly as Theorem 1.1 we can prove that $R_{s}(\mathfrak{p})$ is Noetherian but not Cohen-Macaulay for $\mathfrak{p}=\mathfrak{p}\left(n^{2}, n^{2}+1, n^{2}+n+1\right)$ if $\operatorname{ch} k=p>0$ and if $n=p^{e} \geq 3$ (cf. $\S 3$ ). If we take $n=p=3$, then $\mathfrak{p}=\mathfrak{p}(9,10,13)$. The symbolic Rees algebra $R_{s}(\mathfrak{p})$ for $\mathfrak{p}=\mathfrak{p}(9,10,13)$ remains non-Cohen-Macaulay when $\operatorname{ch} k=2$ (and when $\operatorname{ch} k=7$, too), which we will prove in the last section (cf. Theorem 4.1).

\section{Preliminaries}

Let $(A, \mathfrak{m})$ be a regular local $\operatorname{ring}$ of $\operatorname{dim} A=3$ and $\mathfrak{p}$ a prime ideal of $A$ with $\operatorname{dim} A / \mathfrak{p}=1$. First we shall recall Huneke's criterion for $R_{s}(\mathfrak{p})$ to be Noetherian.

Proposition 2.1 [5]. If there exist $f \in \mathfrak{p}^{(k)}$ and $g \in \mathfrak{p}^{(l)}$ with positive integers $k, l$ such that $l_{A}(A /(f, g, x))=k l \cdot l_{A}(A /(x)+\mathfrak{p})$ for some $x \in \mathfrak{m} \backslash \mathfrak{p}$, then $R_{s}(\mathfrak{p})$ is Noetherian. When the field $A / \mathfrak{m}$ is infinite, the converse is also true. (Here $l_{A}(*)$ stands for the length.)

With this criterion Huneke explored prime ideals $\mathfrak{p}=\mathfrak{p}\left(n_{1}, n_{2}, n_{3}\right)$ and proved that $R_{s}(\mathfrak{p})$ is Noetherian if $\min \left(n_{1}, n_{2}, n_{3}\right)=4$.

In terms of the elements $f$ and $g$ the criterion of Goto, Nishida, and Shimoda is stated as follows, by which they showed $R_{s}(\mathfrak{p}(m, m+1, m+3))$ to be a Gorenstein ring for any $m \geq 1$.

Theorem 2.2 [2]. Let $f$ and $g$ be as in Proposition 2.1. Then the following two conditions are equivalent.

(1) $R_{s}(\mathfrak{p})$ is a Gorenstein ring.

(2) $A /(f, g)+\mathfrak{p}^{(n)}$ are Cohen-Macaulay for all $1 \leq n \leq k+l-2$.

When this is the case, the A-algebra $R_{s}(\mathfrak{p})$ is generated by $\left\{\mathfrak{p}^{(n)} t^{n}\right\}_{1 \leq n \leq k+l-2}$, $f t^{k}$, and $g t^{l}$, and the rings $A /(f)+\mathfrak{p}^{(n)}, A /(g)+\mathfrak{p}^{(n)}$, and $A /(f, g)+\mathfrak{p}^{(n)}$ are Cohen-Macaulay for all $n \geq 1$.

Our proof of Theorem 1.1 consists of two steps. The first step is to find elements $f \in \mathfrak{p}$ and $g \in \mathfrak{p}^{(n+1)}$ satisfying Huneke's condition Proposition 2.1. The second one is to check that $A /(f)+\mathfrak{p}^{(2)}$ cannot be a Cohen-Macaulay ring for that choice of $f$. Then Theorem 2.2 will guarantee the assertion of Theorem 1.1. However for a large class of prime ideals $\mathfrak{p}$ the ring $A /(f)+\mathfrak{p}^{(2)}$ is always non-Cohen-Macaulay for any $f \in \mathfrak{p} \backslash \mathfrak{p}^{(2)}$, and it will make the second step obvious. So in what follows let us give a brief proof of this fact.

We now assume that the ideal $\mathfrak{p}$ is generated by the maximal minors of the 
matrix

$$
\left[\begin{array}{ccc}
X^{\alpha} & Y^{\beta^{\prime}} & Z^{\gamma^{\prime}} \\
Y^{\beta} & Z^{\gamma} & X^{\alpha^{\prime}}
\end{array}\right]
$$

where $X, Y, Z$ is a regular system of parameters for $A$ and $\alpha, \beta, \gamma, \alpha^{\prime}, \beta^{\prime}, \gamma^{\prime}$ are positive integers. We furthermore assume that $\alpha>\alpha^{\prime}, \beta^{\prime}>\beta$, and $\gamma^{\prime}>\gamma$ (this condition is equivalent to saying that $\mathfrak{p}$ is not self-linked, cf. [3]). Let $a=Z^{\gamma+\gamma^{\prime}}-X^{\alpha^{\prime}} Y^{\beta^{\prime}}, b=X^{\alpha+\alpha^{\prime}}-Y^{\beta} Z^{\gamma^{\prime}}$, and $c=Y^{\beta+\beta^{\prime}}-X^{\alpha} Z^{\gamma}$ (hence $\mathfrak{p}=(a, b, c))$. Then because $X^{\alpha} a+Y^{\beta^{\prime}} b+Z^{\gamma^{\prime}} c=Y^{\beta} a+Z^{\gamma} b+X^{\alpha^{\prime}} c=$ 0 , we have $\left(Y^{\beta} a+Z^{\gamma} b\right) \cdot X^{\alpha-\alpha^{\prime}} a=-X^{\alpha} a c=\left(Y^{\beta^{\prime}} b+Z^{\gamma^{\prime}} c\right) \cdot c$, whence $Z^{\gamma}\left(-a b X^{\alpha-\alpha^{\prime}}+c^{2} Z^{\gamma^{\prime}-\gamma}\right)=Y^{\beta}\left(a^{2} X^{\alpha-\alpha^{\prime}}-Y^{\beta^{\prime}-\beta} b c\right)$ so that $Y^{\beta} d_{2}=$ $-a b X^{\alpha-\alpha^{\prime}}+c^{2} Z^{\gamma^{\prime}-\gamma}$ for some $d_{2} \in \mathfrak{p}^{(2)}$. As $d_{2} \equiv Y^{\beta+2 \beta^{\prime}} Z^{\gamma^{\prime}-\gamma} \bmod (X)$, we get the equality

$$
\begin{aligned}
\left(X, d_{2}\right)+\mathfrak{p}^{2}= & \left(X, Y^{2\left(\beta+\beta^{\prime}\right)}, Y^{\beta+2 \beta^{\prime}} Z^{\gamma^{\prime}-\gamma}, Y^{2 \beta+\beta^{\prime}} Z^{\gamma^{\prime}},\right. \\
& \left.Y^{\beta+\beta^{\prime}} Z^{\gamma+\gamma^{\prime}}, Y^{2 \beta} Z^{2 \gamma^{\prime}}, Y^{\beta} Z^{\gamma+2 \gamma^{\prime}}, Z^{2\left(\gamma+\gamma^{\prime}\right.}\right),
\end{aligned}
$$

which yields by an elementary computation that

$$
l_{A}\left(A /\left(X, d_{2}\right)+\mathfrak{p}^{2}\right)=3\left(\beta \gamma+\beta \gamma^{\prime}+\beta^{\prime} \gamma^{\prime}\right) .
$$

On the other hand, as $X$ is a parameter for the Cohen-Macaulay local ring $A / \mathfrak{p}^{(2)}$, the length $l_{A}\left(A /(X)+\mathfrak{p}^{(2)}\right)$ is equal to the multiplicity $e_{X A}\left(A / \mathfrak{p}^{(2)}\right)$ of $A / \mathfrak{p}^{(2)}$ relative to $X$. Therefore because

$$
\begin{aligned}
l_{A}(A /(X)+\mathfrak{p}) & =l_{A}\left(A /\left(X, Y^{\beta+\beta^{\prime}}, Y^{\beta} Z^{\gamma^{\prime}}, Z^{\gamma+\gamma^{\prime}}\right)\right), \\
& =\beta \gamma+\beta \gamma^{\prime}+\beta^{\prime} \gamma^{\prime},
\end{aligned}
$$

we get by the additive formula $[8$, p. 126] of multiplicity that

$$
\begin{aligned}
l_{A}\left(A /(X)+\mathfrak{p}^{(2)}\right) & =l_{A_{\mathfrak{p}}}\left(A_{\mathfrak{p}} / \mathfrak{p}^{2} A_{\mathfrak{p}}\right) \cdot l_{A}(A /(X)+\mathfrak{p}) \\
& =3\left(\beta \gamma+\beta \gamma^{\prime}+\beta^{\prime} \gamma^{\prime}\right) .
\end{aligned}
$$

Hence $l_{A}\left(A /(X)+\mathfrak{p}^{(2)}\right)=l_{A}\left(A /\left(X, d_{2}\right)+\mathfrak{p}^{2}\right)$ so that $(X)+\mathfrak{p}^{(2)}=\left(X, d_{2}\right)+\mathfrak{p}^{2}$. Thus $\mathfrak{p}^{(2)}=\left(d_{2}\right)+\mathfrak{p}^{2}+X \cdot \mathfrak{p}^{(2)}$ and therefore Nakayama's lemma recovers the first assertion of the following lemma. As $\mu_{A}\left(\left(X, d_{2}\right)+\mathfrak{p}^{2}\right)=8$ and as $\mu_{A}\left(\mathfrak{p}^{(2)}\right)=\mu_{A}\left((X)+\mathfrak{p}^{(2)} /(X)\right)$, the second assertion follows from the first. (Here $\mu_{A}(*)$ denotes the number of generators.)

Lemma 2.3 [4]. $\mathfrak{p}^{(2)}=\left(d_{2}\right)+\mathfrak{p}^{2}$ and $\mu_{A}\left(\mathfrak{p}^{(2)}\right)=7$.

The key we need to prove Theorem 1.1 is the following

Proposition 2.4. $A /(f)+\mathfrak{p}^{(2)}$ is not a Cohen-Macaulay ring for any $f \in \mathfrak{p} \backslash \mathfrak{p}^{(2)}$. Proof. Let $f \in \mathfrak{p} \backslash \mathfrak{p}^{(2)}$ and assume that $A /(f)+\mathfrak{p}^{(2)}$ is a Cohen-Macaulay ring. Then because the length $l_{A}\left(A /(X, f)+\mathfrak{p}^{(2)}\right)$ is equal to the multiplicity $e_{X A}\left(A /(f)+\mathfrak{p}^{(2)}\right)$ of $A /(f)+\mathfrak{p}^{(2)}$ relative to the parameter $X$, we get $l_{A}\left(A /(X, f)+\mathfrak{p}^{(2)}\right)=2 e$ by $\left[8\right.$, p. 126] (here $\left.e=l_{A}(A /(X)+\mathfrak{p})\right)$. Let $B=A /(X), I=(X)+\mathfrak{p}) /(X)$, and $J=\left((X)+\mathfrak{p}^{(2)}\right) /(X)$. We put $C=B / J$. Then as $l_{C}(C / f C)=2 e$ and $l_{C}(C)=3 e$, we have $l_{C}(f C)=e$, whence $l_{C}((0): f C)=2 e$ so that $l_{C}(C /(0): f C)=e$. Consequently we must have $J: f B=I$, because $(0): f C \supseteq I / J$ (recall that $J \supseteq I^{2}$ ) and $l_{C}(C /(0): f C)=$ $l_{C}(C /(I / J))=e$. 
Now let $y=Y \bmod (X)$ and $z=Z \bmod (X)$. Then by Lemma 2.3 we see $J=\left(y^{2\left(\beta+\beta^{\prime}\right)}, y^{\beta+2 \beta^{\prime}} z^{\gamma^{\prime}-\gamma}, y^{2 \beta+\beta^{\prime}} z^{\gamma^{\prime}}, y^{\beta+\beta^{\prime}} z^{\gamma+\gamma^{\prime}}, y^{2 \beta} z^{2 \gamma^{\prime}}, y^{\beta} z^{\gamma+2 \gamma^{\prime}}, z^{2\left(\gamma+\gamma^{\prime}\right)}\right)$, while $I=\left(y^{\beta+\beta^{\prime}}, y^{\beta} z^{\gamma^{\prime}}, z^{\gamma+\gamma^{\prime}}\right)$. Therefore a direct computation easily checks that $y^{\beta+\beta^{\prime}-1} z^{\gamma^{\prime}-1} \cdot I \subseteq J$ so that $y^{\beta+\beta^{\prime}-1} z^{\gamma^{\prime}-1} \in J: f B$, whence

$$
y^{\beta+\beta^{\prime}-1} z^{\gamma^{\prime}-1} \in I=\left(y^{\beta+\beta^{\prime}}, y^{\beta} z^{\gamma^{\prime}}, z^{\gamma+\gamma^{\prime}}\right),
$$

which is impossible. Thus $A /(f)+\mathfrak{p}^{(2)}$ cannot be Cohen-Macaulay.

\section{Proof of TheOReM 1.1}

Proof of Theorem 1.1. First notice that the ideal $\mathfrak{p}$ is generated by the maximal minors of the matrix

$$
\left[\begin{array}{ccc}
X^{n} & Y^{n} & Z^{n+1} \\
Y & Z & X
\end{array}\right]
$$

Let $a=Z^{n+2}-X Y^{n}, b=X^{n+1}-Y Z^{n+1}$, and $c=Y^{n+1}-X^{n} Z$. Then as $X^{n} a+Y^{n} b+Z^{n+1} c=Y a+Z b+X c=0$, we have $Y^{n} a^{n}+Z^{n} b^{n}+X^{n} c^{n}=0$, whence

$$
\left(Z^{n} b^{n}+X^{n} c^{n}\right) b=-Y^{n} a^{n} b=\left(X^{n} a+Z^{n+1} c\right) a^{n} .
$$

Thus $X^{n}\left(a^{n+1}-b c^{n}\right)=Z^{n}\left(b^{n+1}-a^{n} c Z\right)$ and so $X^{n} e_{n+1}=b^{n+1}-a^{n} c Z$ for some $e_{n+1} \in \mathfrak{p}^{(n+1)}$. Because $c \equiv Y^{n+1} \bmod (Z)$ and $e_{n+1} \equiv X^{n^{2}+n+1} \bmod (Z)$, we get

$$
l_{A}\left(A /\left(c, e_{n+1}, Z\right)\right)=1 \cdot(n+1) \cdot l_{A}(A /(Z)+\mathfrak{p})=(n+1)\left(n^{2}+n+1\right)
$$

whence $R_{s}(\mathfrak{p})$ is, by Proposition 2.1, Noetherian. As $A /(c)+\mathfrak{p}^{(2)}$ is, by Proposition 2.4 , not a Cohen-Macaulay ring, we conclude by Theorem 2.2 that $R_{s}(\mathfrak{p})$ is non-Cohen-Macaulay.

Corollary 3.1 [2]. $R_{s}(\mathfrak{p})$ is not a Cohen-Macaulay ring for $\mathfrak{p}=\mathfrak{p}(7,9,10)$ if $\operatorname{ch} k=2$.

Theorem 3.2. Let $p$ be a prime number and $n=p^{e}$ with $e \geq 1$. Then $R_{s}(\mathfrak{p})$ is Noetherian but not Cohen-Macaulay for $\mathfrak{p}=\mathfrak{p}\left(n^{2}, n^{2}+1, n^{2}+n+1\right)$ if $\operatorname{ch} k=p$ and $n \geq 3$.

Proof. The ideal $\mathfrak{p}$ is generated by the maximal minors of the matrix

$$
\left[\begin{array}{ccc}
X^{n} & Y^{n} & Z^{n-1} \\
Y & Z & X
\end{array}\right]
$$

Let $a=Z^{n}-X Y^{n}, b=X^{n+1}-Y Z^{n-1}$, and $c=Y^{n+1}-X^{n} Z$. Then $X^{n} a+Y^{n} b+Z^{n-1} c=Y a+Z b+X c=0$ so that $Y^{n} a^{n}+Z^{n} b^{n}+X^{n} c^{n}=0$, too. Similarly as in the proof of Theorem 1.1 we compare two expressions of $Y^{n} a^{n} b$ to get that $X^{n} e_{n+1}=b^{n+1} Z-a^{n} c$ for some $e_{n+1} \in \mathfrak{p}^{(n+1)}$. As $e_{n+1} \equiv(-1)^{n} Y^{n^{2}+n+1} \bmod (Z)$, we have

$$
l_{A}\left(A /\left(b, e_{n+1}, Z\right)\right)=1 \cdot(n+1) \cdot l_{A}(A /(Z)+\mathfrak{p})=(n+1)\left(n^{2}+n+1\right),
$$

whence $R_{s}(\mathfrak{p})$ is Noetherian by Proposition 2.1 but not Cohen-Macaulay by Theorem 2.2 and Proposition 2.4. 
4. The NON-COHEN-MACaulayness of $R_{s}(\mathfrak{p})$

FOR $\mathfrak{p}=\mathfrak{p}(9,10,13)$ IN THE CASE OF $\operatorname{ch} k=2$

Let $\mathfrak{p}=\mathfrak{p}(9,10,13)$. Then by Theorem $3.2 R_{s}(\mathfrak{p})$ is Noetherian but not Cohen-Macaulay if $\operatorname{ch} k=3$. The same is true when $\operatorname{ch} k=2$ too, as we shall prove in the following

Theorem 4.1. Suppose that $\operatorname{ch} k=2$. Then $R_{s}(\mathfrak{p})$ is Noetherian but non-CohenMacaulay for $\mathfrak{p}=\mathfrak{p}(9,10,13)$.

We divide the proof of Theorem 4.1 into a few parts. First notice that $\mathfrak{p}$ is generated by the maximal minors of the matrix

$$
\left[\begin{array}{ccc}
X^{3} & Y^{3} & Z^{2} \\
Y & Z & X
\end{array}\right]
$$

Let $a=Z^{3}+X Y^{3}, b=X^{4}+Y Z^{2}$, and $c=Y^{4}+X^{3} Z$. Hence $\mathfrak{p}=(a, b, c)$. We put

$$
\begin{gathered}
d_{2}=Y^{7} Z+X^{2} Z^{5}+X^{3} Y^{3} Z^{2}+X^{7} Y^{2} \\
d_{3}=Y^{10}+X Z^{7}+X^{2} Y^{3} Z^{4}+X^{3} Y^{6} Z+X^{6} Y^{2} Z^{2}+X^{10} Y, \\
d_{4}=Z^{11}+Y^{13} Z+X^{3} Y^{9} Z^{2}+X^{6} Y^{5} Z^{3} \\
+X^{7} Y^{8}+X^{9} Y Z^{4}+X^{10} Y^{4} Z+X^{13} Z^{2}, \\
d_{7}=Z^{19}+X Y^{3} Z^{16}+X^{3} Y^{22}+X^{4} Y^{12} Z^{7}+X^{5} Y^{2} Z^{14}+X^{5} Y^{15} Z^{4} \\
+X^{7} Y^{8} Z^{8}+X^{8} Y^{18} Z+X^{9} Y Z^{12}+X^{9} Y^{14} Z^{2}+X^{10} Y^{4} Z^{9} \\
+X^{13} Y^{13}+X^{15} Y^{6} Z^{4}+X^{18} Y^{2} Z^{5}+X^{23} Y^{4}+X^{26} Z .
\end{gathered}
$$

Then a direct calculation checks the equalities

$$
\begin{array}{cl}
X d_{2}=b^{2} Y^{2}+a c Z, & Z^{2} d_{3}=a^{3} X+b c^{2} Y, \\
Z d_{4}=a^{4}+b c^{3}, & X d_{7}=a^{4} d_{3}+b^{6} c
\end{array}
$$

so that

Lemma 4.6. $d_{n} \in \mathfrak{p}^{(n)}$ for $n=2,3,4$, and 7 .

As $b^{10}+d_{3} d_{7} Z \equiv 0 \bmod (X)$, we have $X d_{10}=b^{10}+d_{3} d_{7} Z$ with $d_{10} \in \mathfrak{p}^{(10)}$. Notice that $b \equiv Y Z^{2}, d_{3} \equiv Y^{10}+X Z^{7}$, and $d_{7} \equiv Z^{19}+X Y^{3} Z^{16} \bmod \left(X^{2}\right)$ and we get

$$
d_{10} \equiv Z^{27}+Y^{13} Z^{17} \bmod (X) .
$$

Thus $R_{s}(p)$ is Noetherian by Proposition 2.1 , because

$$
l_{A}\left(A /\left(d_{3}, d_{10}, X\right)\right)=l_{A}\left(A /\left(X, Y^{10}, Z^{27}\right)\right)=270=3 \cdot 10 \cdot l_{A}(A /(X)+\mathfrak{p}) \text {. }
$$

To see that $R_{s}(\mathfrak{p})$ is not Cohen-Macaulay we need the following

Proposition 4.8. (1) $\mathfrak{p}^{(2)}=\left(d_{2}\right)+\mathfrak{p}^{2}$.

(2) $\mathfrak{p}^{(3)}=\left(d_{3}\right)+\mathfrak{p}^{3}$.

(3) $\mathfrak{p}^{(4)}=\left(d_{4}\right)+d_{3} \mathfrak{p}+\mathfrak{p}^{4}$.

(4) $\mathfrak{p}^{(5)}=\mathfrak{p p}^{(4)}$.

(5) $\mathfrak{p}^{(6)}=\left(d_{3}^{2}\right)+\mathfrak{p p}^{(5)}$. 
(6) $\mathfrak{p}^{(7)}=\left(d_{7}\right)+\left(d_{3} d_{4}\right)+\mathfrak{p p}^{(6)}$.

(7) $\mathfrak{p}^{(8)}=\mathfrak{p p}^{(7)}$.

(8) $\mathfrak{p}^{(9)}=\left(d_{3}^{2}\right)+d_{7} \mathfrak{p}^{(2)}+d_{3} d_{4} \mathfrak{p}^{(2)}+d_{3}^{2} \mathfrak{p}^{3}+d_{4} \mathfrak{p}^{5}+d_{3} \mathfrak{p}^{6}+\mathfrak{p}^{9}$.

(9) $\mathfrak{p}^{(10)}=\left(d_{10}, d_{3} d_{7}, d_{3}^{2} d_{4}\right)+\mathfrak{p p}^{(9)}$.

(10) $\mathfrak{p}^{(11)}=\mathfrak{p p}^{(10)}$.

Proof. Let $I_{n}(2 \leq n \leq 11)$ denote the ideals on the right-hand side of the above equalities. Then

$$
l_{A}\left(A /(X)+I_{n}\right) \geq l_{A}\left(A /(X)+\mathfrak{p}^{(n)}\right)=e_{X A}\left(A / \mathfrak{p}^{(n)}\right)=9 \cdot(n+1) n / 2
$$

for each $2 \leq n \leq 11$ (to see the last equality use the additive formula [8, $\mathrm{p}$. 126] of multiplicity). Hence if we can show the inequality $l_{A}\left(A /(X)+I_{n}\right) \leq$ $9(n+1) n / 2$, then we naturally have $(X)+I_{n}+(X)+\mathfrak{p}^{(n)}$ so that $\mathfrak{p}^{(n)}=I_{n}$ by Nakayama's lemma (cf. proof of Lemma 2.3). Checking of the inequalities $l_{A}\left(A /(X)+I_{n}\right) \leq 9(n+1) n / 2$ is direct, which we leave to the readers.

Proposition 4.9. The ring $A /\left(d_{3}\right)+\mathfrak{p}^{(n)}$ is Cohen-Macaulay for each $1 \leq n \leq 11$. Hence

$$
R_{s}(\mathfrak{p})=A\left[a t, b t, c t, d_{2} t^{2}, d_{3} t^{3}, d_{4} t^{4}, d_{7} t^{7}, d_{10} t^{10}\right] .
$$

Proof. The second assertion follows from the first (cf. [2, (3.7)(4)]). To check the first assertion we may assume $4 \leq n \leq 11$. It suffices to show

$$
l_{A}\left(A /\left(X, d_{3}\right)+\mathfrak{p}^{(n)}\right) \leq e_{X A}\left(A /\left(d_{3}\right)+\mathfrak{p}^{(n)}\right) .
$$

Notice that $e_{X A}\left(A /\left(d_{3}\right)+\mathfrak{p}^{(n)}\right)=9 \cdot l_{A_{\mathfrak{p}}}\left(A_{\mathfrak{p}} / d_{3} A_{\mathfrak{p}}+\mathfrak{p}^{n} A_{\mathfrak{p}}\right)$ by [8, p. 126], and we get

$$
e_{X A}\left(A /\left(d_{3}\right)+\mathfrak{p}^{(n)}\right)=27(n-1)
$$

for $4 \leq n \leq 11$. Because we have already known by Proposition 4.8 the ideals $\left(X, d_{3}\right)+\mathfrak{p}^{(n)}$ explicitly, checking of the inequalities $l_{A}\left(A /\left(X, d_{3}\right)+\mathfrak{p}^{(n)}\right) \leq$ $27(n-1)$ for $4 \leq n \leq 11$ is elementary, whose detail is left to the readers.

Let us finish the proof of Theorem 4.1. By Theorem 2.2 it suffices to see that $A /\left(d_{3}, d_{10}\right)+\mathfrak{p}^{(11)}$ is not a Cohen-Macaulay ring. By Proposition 4.8 we have

$$
\left(X, d_{3}, d_{10}\right)+\mathfrak{p}^{(11)}=(X)+\left(Y^{10}, Y^{9} Z^{24}, Y^{7} Z^{25}, Y^{6} Z^{26}, Z^{27}\right)
$$

so that $l_{A}\left(A /\left(X, d_{3}, d_{10}\right)+\mathfrak{p}^{(11)}\right)=262$, while

$$
e_{X A}\left(A /\left(d_{3}, d_{10}\right)+\mathfrak{p}^{(11)}\right)=9 \cdot l_{A_{\mathfrak{p}}}\left(A_{\mathfrak{p}} /\left(d_{3}, d_{10}\right) A_{\mathfrak{p}}+\mathfrak{p}^{11} A_{\mathfrak{p}}\right)=9 \cdot 29=261
$$

(recall that $d_{3}, d_{10}$ forms a super regular sequence in $A_{\mathfrak{p}}$, cf. [2, (3.1)(3)]). Hence

$$
l_{A}\left(A /\left(X, d_{3}, d_{10}\right)+\mathfrak{p}^{(11)}\right)>e_{X A}\left(A /\left(d_{3}, d_{10}\right)+\mathfrak{p}^{(11)}\right)
$$

and so $A /\left(d_{3}, d_{10}\right)+\mathfrak{p}^{(11)}$ cannot be Cohen-Macaulay. This completes the proof of Theorem 4.1.

Remark 4.10. Let $M$ be the unique graded maximal ideal of $R_{s}(\mathfrak{p})$. Then we have

$$
\operatorname{dim} R_{s}(\mathfrak{p})_{M}=4 \text { and } \operatorname{depth} R_{s}(\mathfrak{p})_{M}=3
$$

for the symbolic Rees algebras $R_{s}(\mathfrak{p})$ dealt with in Theorems 1.1, 3.2, and 4.1 (cf. [2, (2.1), (3.7)(3)]). 


\section{REFERENCES}

1. R. C. Cowsik, Symbolic powers and the number of defining equations, Algebra and Its Applications, Lecture Notes in Pure and Appl. Math., vol. 91, Dekker, New York, 1985, pp. 13-14.

2. S. Goto, K. Nishida, and Y. Shimoda, The Gorensteinness of symbolic Rees algebras for space curves, J. Math. Soc. Japan 43 (1991), 465-481.

3. J. Herzog and B. Ulrich, Self-linked curve singularities, Nagoya Math. J. 120 (1990), 129153.

4. C. Huneke, The primary components of and integral closures of ideals in 3-dimensional regular local rings, Math. Ann. 275 (1986), 617-635.

5. __ Hilbert functions and symbolic powers, Michigan Math. J. 34 (1987), 293-318.

6. M. Nagata, On the fourteenth problem of Hilbert, Proc. Internat. Congr. Math. (1958), Cambridge Univ. Press, London and New York, 1960.

7. P. Roberts, A prime ideal in a polynomial ring whose symbolic blow-up is not Noetherian, Proc. Amer. Math. Soc. 94 (1985), 589-592.

8. J.-P. Serré, Algèbre locale: multilicités, 3rd ed., Lecture Notes in Math., vol. 11, SpringerVerlag, Berlin and New York, 1975.

9. A. Simis and N. V. Trung, The divisor class group of ordinary and symmetric blow-ups, Math. Z. 198 (1988), 479-491.

10. W. V. Vasconcelos, Symmetric algebras and factoriality, Math. Sci. Res. Inst. Publ., vol. 15, Springer, New York, 1989, pp. 467-496.

Department of Mathematics, Tokyo Christian Women's University, Zenpuku-Ji 2-6-1, SUGINAMI-KU, TOKYO 167 , JAPAN

Department of Mathematics, School of Science and Technology, Meiji University, Higashimita 1-1-1, TAMA-KU, KaWASAKI-SHI 214, JaPAN

E-mail address: goto@math.meiji.ac.jp 